\title{
PERFORMANCE ASSESSMENT OF A RECENT CHANGE DETECTION METHOD FOR HOMOGENEOUS AND HETEROGENEOUS IMAGES
}

\author{
Jorge Prendes ${ }^{1,3,5}$, Marie Chabert ${ }^{1,2}$, Frédéric Pascal ${ }^{3}$, Alain Giros ${ }^{4}$, Jean-Yves Tourneret ${ }^{1,2}$ \\ 1: TéSA Laboratory, Toulouse, France \\ 2: University of Toulouse, INP/ENSEEIHT - IRIT, Toulouse, France \\ 3: Supélec - SONDRA, Gif-sur-Yvette, France \\ 4: CNES (French Space Agency), Toulouse, France \\ 5: jorge.prendes@tesa.prd.fr
}

\begin{abstract}
Résumé
Un modèle statistique pour détecter des changements dans les images de télédétection a récemment été proposé dans (Prendes et al., 2014, 2015). Ce modèle, étant très général, peut être utilisé pour des images homogènes acquises par le même type de capteurs (par exemple, deux images optiques des satellites Pléiades, éventuellement avec des conditions d'acquisition différentes), mais aussi pour des images hétérogènes acquises par des capteurs de natures différentes (par exemple, une image optique acquise par un satellite Pléiade et une image radar à synthèse d'ouverture (RSA) acquise par un satellite TerraSAR-X). Ce modèle suppose que chaque pixel est distribué selon un mélange de densités de probabilité liées aux propriétés du bruit ainsi qu' aux réponses en intensité renvoyées par la scène réelle. Les paramètres du modèle statistique sont estimés en utilisant l'algorithme classique d'expectation-maximization (EM). Ensuite, ces paramètres estimés sont utilisés afin d'inférer les relations entre les images d'intérêt, en utilisant une stratégie d'apprentissage sur des variétés. Ces relations sont pertinentes pour de nombreuses applications du traitement d'images, en particulier celles qui requièrent une mesure de similarité (par exemple, les problèmes de détection de changements dans des images ou encore de recalage d'images). L'objectif principal de cet article est d'évaluer les performances d'une méthode de détection de changements basée sur cette stratégie d'apprentissage de variétés initialement introduite dans (Prendes et al., 2014, 2015). Ces performances sont analysées grâce à des paires d'images optiques réelles et des paires d'images optiques/RSA Pléiades.
\end{abstract}

Mots clés : Télédétection, images hétérogènes, Pléiades, SAR, détection des changements, mesure de similarité

\begin{abstract}
A statistical model for detecting changes in remote sensing images has recently been proposed in (Prendes et al., 2014, 2015). This model is sufficiently general to be used for homogeneous images acquired by the same kind of sensors (e.g., two optical images from Pléiades satellites, possibly with different acquisition conditions), and for heterogeneous images acquired by different sensors (e.g., an optical image acquired from a Pléiades satellite and a synthetic aperture radar (SAR) image acquired from a TerraSAR-X satellite). This model assumes that each pixel is distributed according to a mixture of distributions depending on the noise properties and on the sensor intensity responses to the actual scene. The parameters of the resulting statistical model can be estimated by using the classical expectation-maximization (EM) algorithm. The estimated parameters are finally used to learn the relationships between the images of interest, via a manifold learning strategy. These relationships are relevant for many image processing applications, particularly those requiring a similarity measure (e.g., image change detection and image registration). The main objective of this paper is to evaluate the performance of a change detection method based on this manifold learning strategy initially introduced in (Prendes et al., 2014, 2015). This performance is evaluated by using results obtained with pairs of real optical images acquired from Pléiades satellites and pairs of optical and SAR images.
\end{abstract}

Keywords : Remote sensing, heterogeneous images, Pléiades, SAR, change detection, similarity measure

\section{Introduction}

Current remote sensing imagery exploits many different technologies. Various kinds of sensors with different pros and cons (spatial resolution, noise properties, acquisition conditions, etc.) provide complementary information about the observed scene. In particular, optical remote sensing imagery provided by Pléiades satellites offers a high spatial resolution. Synthetic aperture radar
(SAR) images can be acquired in the presence of clouds or even during the night (Schowengerdt, 2006; Curlander and McDonough, 1991; Carrara et al., 1995). Hyperspectral images (Schowengerdt, 2006) can acquire hundreds of different spectral bands for material characterization.

Exploiting the complementary properties of remote sensing images is important in many practical applica- 
tions. A classical application is the detection of changes between optical images available in a database and a newly acquired SAR image. A change detector able to deal with heterogeneous ${ }^{1}$ images can be particularly useful after natural disasters such as floodings, volcano eruptions or earthquakes (Uprety and Yamazaki, 2012). Indeed, in case of emergency, we may have to compare quickly available SAR images and optical images contained in a database, detect the affected areas in order to build an efficient action plan. Change detection between heterogeneous images is also interesting for other applications including urban growth tracking (Storie et al., 2012; Tison et al., 2004), plantation monitoring, and urban database updating (Poulain et al., 2010).

A new change detection strategy adapted to homogeneous as well as heterogeneous images was recently proposed in (Prendes et al., 2014, 2015). The main goal of this paper is to evaluate the performance of this strategy for detecting changes between pairs of real homogeneous optical Pléiades images and pairs of heterogeneous optical and SAR images.

The paper is organized as follows: Sections 2 and 3 review the image model and similarity measure introduced in (Prendes et al., 2014, 2015) for change detection, with a special emphasis on Pléiades images. Simulation results conducted on different datasets are shown in Section 4. These results allow the performance of the change detection method studied in (Prendes et al., $2014,2015)$ to be assessed. Conclusions and future work are finally presented in Section 5 .

\section{Joint Statistical Model for Pixel Intensities}

This section summarizes the main elements of the image generation model in (Prendes et al., 2014, 2015). A pixel intensity is modeled as a ground truth corrupted by some measurement noise.

A usual approach for statistical change detection is based on analyzing the contents of a sliding window, as opposed to a pixel by pixel comparison. This approach is illustrated in Fig. 1. To determine whether a pixel has changed from one image to another, we consider an analysis window in both images centered on that pixel. A similarity measure is then computed to determine the similarity between the images within this window, resulting in a real value $d \in \mathbb{R}$. A hypothesis test is finally constructed for $d$ to determine the presence or absence of a change in the window of interest. This process is repeated for each image pixel in the image in order to build a change mask.

\subsection{Homogeneous windows}

A window is homogeneous when the ground truth is constant for all its pixels. It is the case when a single

\footnotetext{
${ }^{1}$ Homogeneous images have been acquired by two different sensors of the same kind (e.g., two optical images or two SAR images) contrary to heterogeneous images (e.g., one optical and one SAR image).
}

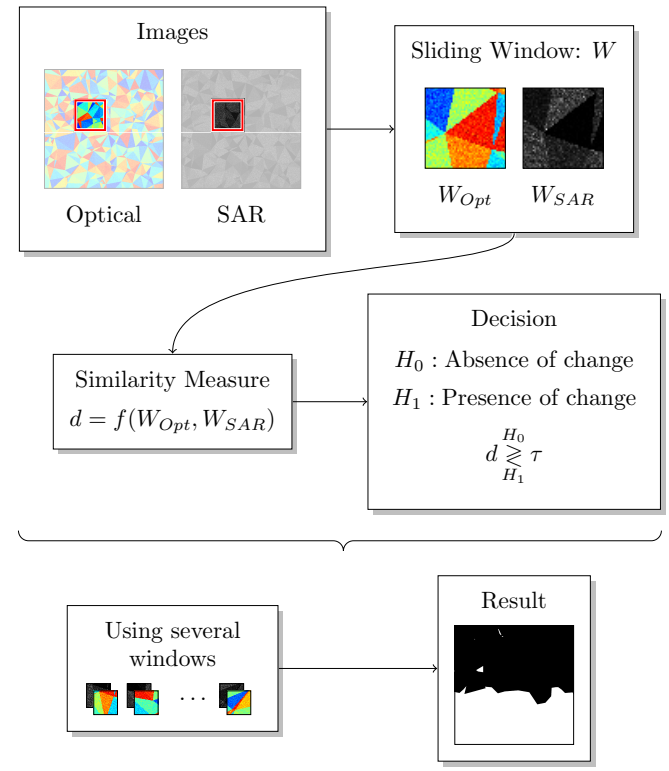

Figure 1: Change detection strategy using a similarity measure.

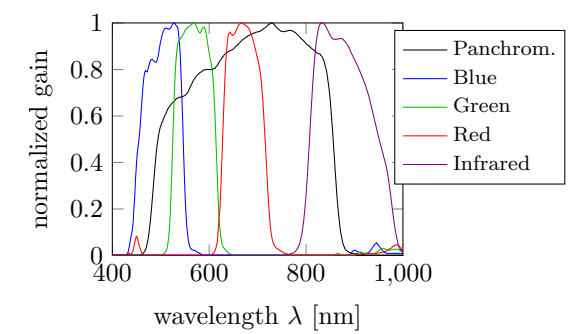

Figure 2: Spectral bands of the Pléiades sensors.

object is included in the window, such as a red roof, a field cultivated with a specific plantation, a road, etc.

As explained in (Prendes et al., 2014, 2015), the pixel intensity of an optical image, $I_{\text {opt }}$, can be modeled as a ground truth intensity, $G_{\text {Opt }}$, corrupted by additive zeromean Gaussian noise $\nu_{\text {Opt }}$, i.e.,

$$
I_{\text {Opt }}=G_{\text {Opt }}+\nu_{\text {Opt }} \text {. }
$$

As optical Pléiades image are composed of 4 different images associated with 4 different spectral bands, $I_{\mathrm{Opt}}$ and $G_{\mathrm{Opt}}$ are 4-dimensional vectors denoted as $I_{\mathrm{Opt}}=$ $\left[I_{\mathrm{IR}}, I_{\mathrm{R}}, I_{\mathrm{G}}, I_{\mathrm{B}}\right]$ and $G_{\mathrm{Opt}}=\left[G_{\mathrm{IR}}, G_{\mathrm{R}}, G_{\mathrm{G}}, G_{\mathrm{B}}\right]$.

On the other hand, SAR images can be modeled as a ground truth intensity corrupted with a multiplicative speckle noise distributed according to a gamma distribution, i.e.,

$$
I_{\mathrm{SAR}}=G_{\mathrm{SAR}} \times \nu_{\mathrm{SAR}} .
$$

From these models, the pixel intensities of optical and SAR images conditionally on the ground truth intensity can be expressed as

$$
\begin{aligned}
I_{\text {Opt }} \mid G_{\text {Opt }} & \sim \mathcal{N}\left(G_{\text {Opt }}, \sigma_{\text {Opt }}^{2}\right), \\
I_{\text {SAR }} \mid G_{\text {SAR }} & \sim \Gamma\left(L_{\text {SAR }}, G_{\text {SAR }} L_{\text {SAR }}^{-1}\right),
\end{aligned}
$$

where $\sigma_{\mathrm{Opt}}$ is related to the signal to noise ratio of the optical sensor, and $L_{\mathrm{SAR}}$ is related to the number of looks of the SAR image.

The ground truth intensities $G_{\text {Opt }}$ and $G_{\text {SAR }}$ both depend on the object to be imaged. We denote as $P$ the physical properties of this object, e.g., its reflectivity spec- 
trum. Given the spectral response of the Pléiades satellite sensors (shown in Fig. 2), the corresponding ground truth intensity for each spectral band can be obtained. Denote as $T_{S}(\cdot)$ the operation transforming the object $P$ into the ground truth for the sensor $S$, i.e., such that

$$
G_{S}=T_{S}(P) \text {. }
$$

For instance, considering the Pléiades blue spectral band filter $F_{\mathrm{B}}(\lambda)$ and the material reflectivity $R(P, \lambda)$, the blue band ground truth $G_{\mathrm{B}}$ can be expressed as follows

$$
G_{\mathrm{B}}=T_{\mathrm{B}}(P)=\int_{-\infty}^{\infty} F_{\mathrm{B}}(\lambda) R(P, \lambda) d \lambda
$$

and equivalently for the other spectral bands.

The pixel intensities contained in a homogeneous window are statistically independent given the ground truth. As a consequence, their joint distribution conditionally on $P$ is simply the product of Eqs. (3) and (4), yielding

$$
p\left(I_{\mathrm{Opt}}, I_{\mathrm{SAR}} \mid P\right)=p\left(I_{\mathrm{Opt}} \mid T_{\mathrm{Opt}}(P)\right) \times p\left(I_{\mathrm{SAR}} \mid T_{\mathrm{SAR}}(P)\right) \text {. }
$$

\subsection{Heterogeneous windows}

An image generally consists of many heterogeneous windows containing different objects. It is interesting to note that these heterogeneous windows are more appropriate for statistical change detection. Indeed, the methods based on a statistical dependency (e.g., based on the correlation coefficient or the mutual information) fail when homogeneous areas are considered. This can be explained by Eq. (7), which states that the pixel intensities contained in a homogeneous windows are statistically independent. In (Prendes et al., 2014, 2015) we assumed that different objects contained within the analysis windows lead to different homogeneous regions. Thus, a finite number $K$ of objects is supposed to be present within any analysis window $W$, each one with its own physical properties $P_{k}$ with $1 \leq k \leq K$. The probability of finding a particular property $P_{k}$ in $W$ is related to the area of the window covered by the object with property $P_{k}$, i.e.,

$$
p(P \mid W)=\sum_{k=1}^{K} w_{k} \delta\left(P-P_{k}\right)
$$

where $\delta(\cdot)$ represents the Dirac delta function. From Eqs. (7) and (8), $p\left(I_{\mathrm{Opt}}, I_{\mathrm{SAR}} \mid W\right)$ can be obtained by integrating out $P$

$$
\begin{aligned}
p\left(I_{\mathrm{Opt}}, I_{\mathrm{SAR}} \mid W\right) & =\int p\left(I_{\mathrm{Opt}}, I_{\mathrm{SAR}} \mid P, W\right) p(P \mid W) d P \\
& =\sum_{k=1}^{K} w_{k} p\left(I_{\mathrm{Opt}}, I_{\mathrm{SAR}} \mid P_{k}\right) .
\end{aligned}
$$

Eq. (10) shows that the pixel intensity within a heterogeneous analysis window is distributed according to a mixture distribution. The components of this mixture belong to a family of distributions defined by the sensor type. The parameter vector associated with the $k$ th component of this mixture, denoted as $\theta_{k}$, depends on the physical properties $P_{k}$ of that component. Using a slightly modified version of the expectation maximization (EM) algorithm as described in (Figueiredo and Jain, 2002; Prendes et al., 2014, 2015), we can estimate the mixture parameters, namely, the number of components $K$ and their corresponding parameters $\theta_{k}$ with $1 \leq k \leq K$.

\section{Similarity Measure}

The model presented in Section 2 can be used to define a similarity measure for change detection (Prendes et al., 2014, 2015) . This section recalls the different steps required for the construction of this measure for optical and SAR images. We assume that the vector $\boldsymbol{v}(P)=\left[T_{\mathrm{Opt}}(P), T_{\mathrm{SAR}}(P)\right]$ belongs to a manifold in a $D$ dimensional space in the absence of change between the two images (this assumption will be justified later), describing the link between the different sensors. For instance, in the case of an optical Pléiades image and a SAR image, the vector $\boldsymbol{v}(P)$ is defined as

$$
\boldsymbol{v}(P)=\left[T_{\mathrm{R}}(P), T_{\mathrm{R}}(P), T_{\mathrm{G}}(P), T_{\mathrm{B}}(P), T_{\mathrm{SAR}}(P)\right]
$$

and lives in a 5-dimensional space. Of course, the transformations $T_{s}(\cdot)$ are unknown or difficult to obtain analytically in practical applications. The proposed approach consists of estimating this manifold using unchanged areas in the the two images as learning data.

Fig. 3 illustrates this manifold learning strategy for synthetic data, where the manifold is defined by the relationship $T_{\text {SAR }}(P)=T_{\text {Opt }}(P) \times\left[1-T_{\text {Opt }}(P)\right]$. More precisely, given an analysis window $W$, and its corresponding optical and SAR components $W_{\text {Opt }}$ and $W_{\text {SAR }}$, we propose to estimate the parameters of the mixture distribution (10), i.e., the number of components $K$, the components weight $w_{k}$, and the components parameters $\boldsymbol{\theta}_{k}$, from training data belonging to unchanged areas of the two images (supervised learning). These estimated parameters are then used to estimate $K$ realizations of $\boldsymbol{v}(P)$ (associated with the ground truth of each image), denoted $\left.\widehat{\boldsymbol{v}}\left(P_{k}\right)\right|_{k=1} ^{K}$, one for each component of the mixture distribution. Repeating this process for various analysis windows (associated with unchanged areas), different estimations of $\boldsymbol{v}(P)$ are obtained and can be used to estimate the expected manifold.

On the other hand, a vector $\widehat{\boldsymbol{v}}\left(P_{k}\right)$ associated with a changed area is expected to lie outside of the learned manifold. Using this property, we have introduced in (Prendes et al., 2014, 2015) a similarity measure based on the estimated distance between $\widehat{\boldsymbol{v}}\left(P_{k}\right)$ and the manifold. More precisely, we used the estimated density associated with the vectors $\widehat{\boldsymbol{v}}\left(P_{k}\right)$ in unchanged areas resulting from the learning stage as our new similarity measure for change detection. This density can be easily learned using different methods, as discussed in (Prendes et al., 2014, 2015).

\section{Simulation Results}

This section evaluates the performance of the change detection strategy described in Section 3 for different datasets involving Pléiades images. The performance is evaluated by means of the receiver operating characteristic (ROC) curves (Peterson et al., 1954) that are classical performance measures for detection problems. Dif- 

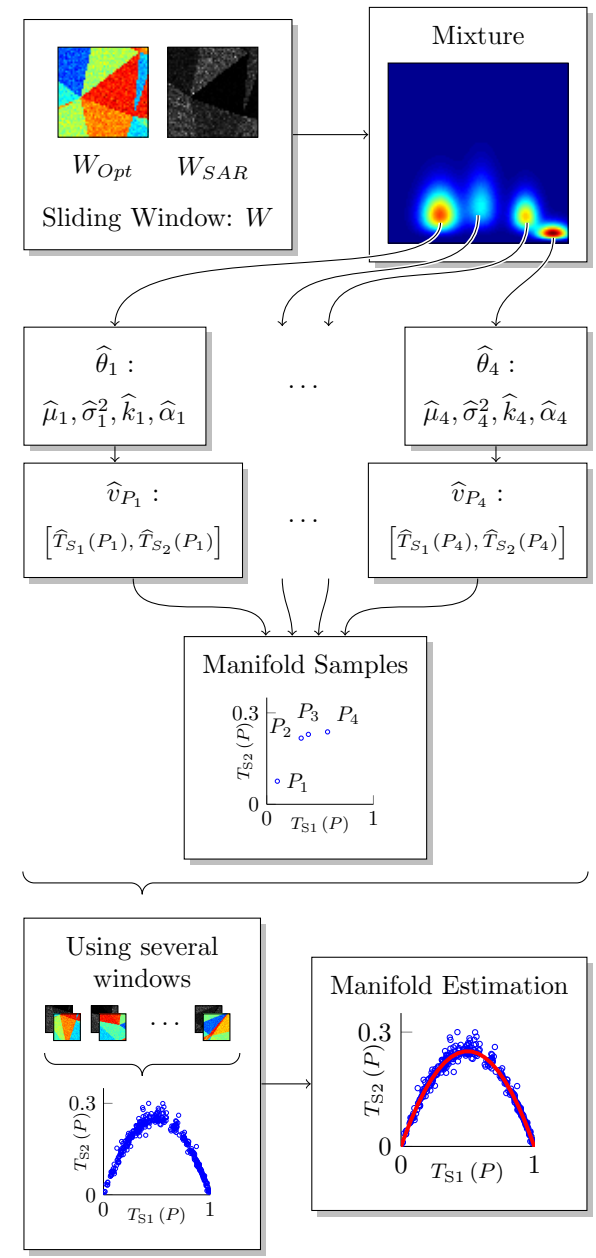

Figure 3: Manifold estimation for synthetic data.

ferent datasets are used to assess the detection performance in a variety of situations involving homogeneous and heterogeneous images. A comparison with other state-of-the-art change detection methods is also performed.

\subsection{Optical images from the same sensor}

The first test is conducted on a set of two Pléiades images captured from sensors with the same specifications. The images presented in Figs. 4(a) and 4(b) show the test images corresponding to an area located in the south of the city of Toulouse in France, before and after a building work. Fig. 4(c) shows the change mask used as the ground truth for this experiment. Note that this change mask construction as well as the image registration procedure were conducted by a photo interpreter. The multispectral images, with a resolution of $2 \mathrm{~m}$, were pan-sharpened using the panchromatic image with a resolution of $50 \mathrm{~cm}$. This step is required since all images have to be co-registered. For simplicity, a projection substitution pansharpening algorithm (Thomas et al., 2008) was implemented, where each multi-spectral component was up-sampled to match the resolution of the panchromatic component $(50 \mathrm{~cm})$. Moreover, its intensity (computed using an L-2 norm) was then replaced by the panchromatic component to produce a multi-spectral (4 spectral bands) image with a $50 \mathrm{~cm}$ resolution. The change map

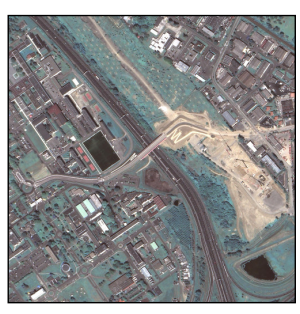

(a) Pléiades - May 2012

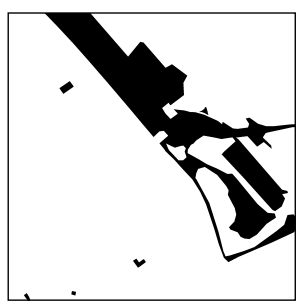

(c) Change mask

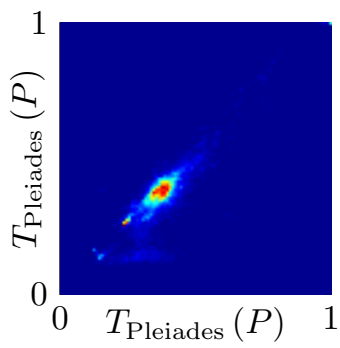

(e) 2D projected manifold

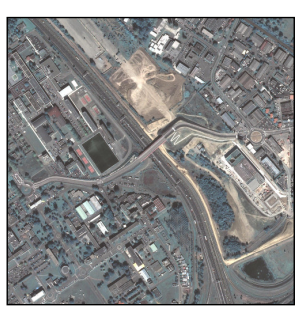

(b) Pléiades - Sep. 2013

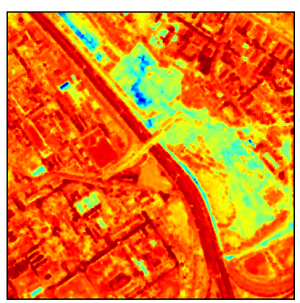

(d) Computed similarity

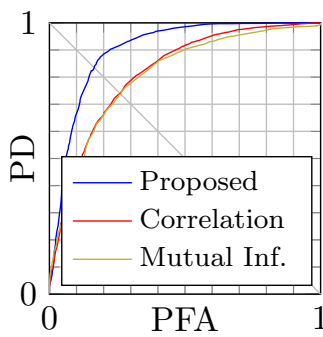

(f) Detection performance
Figure 4: Optical images from homogeneous sensors before (a) and after (b) a building work, with the corresponding change mask (c) and detector output map (d) (where red represents high similarity, and blue represents low similarity). The obtained estimated manifold (e) (where red represents high density, and blue represents low density) and detector performance (f).

obtained with the proposed detector is shown in Fig. 4(d). Red areas represent high similarity between the two images, while blue areas correspond to low similarity. The map was obtained with a moving window of size $40 \times 40$ pixels, covering an area of $20 \times 20 \mathrm{~m}^{2}$. The main changes due to building works can be observed in this map.

Fig. 4(e) shows a 2D projection of the 8-dimensional estimated manifold. Since both images have been acquired by homogeneous sensors, we expect the manifold to reveal a linear relationship between the two variables (Prendes et al., 2014, 2015). However, minor nonlinear effects can also be observed which can be attributed to different illumination conditions producing different shadow patterns, as well as some sensor saturation.

As the threshold used to produce the change detection map is strongly application dependent, we compute ROC curves to evaluate the performance of the proposed detector and to compare it with respect to other stateof-the-art methods. For the first dataset (two homogeneous Pléiades images), Fig. 4(f) shows a comparison with the correlation coefficient and the mutual information, both measuring the statistical dependency between two random variables. The correlation coefficient mea- 


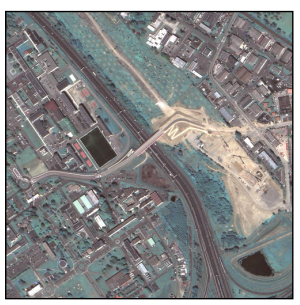

(a) Pléiades - May 2012

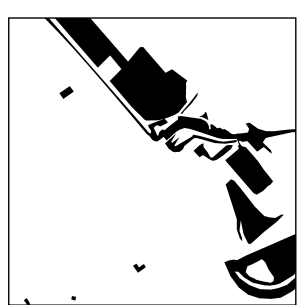

(c) Change mask

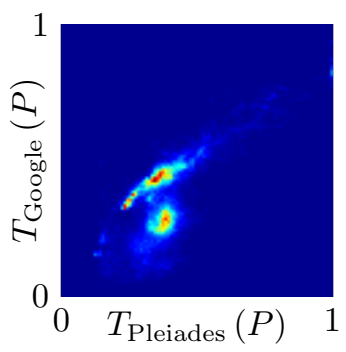

(e) 2D projected manifold

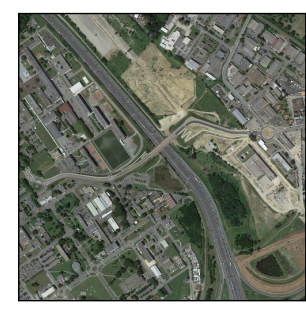

(b) Google Earth - July 2013

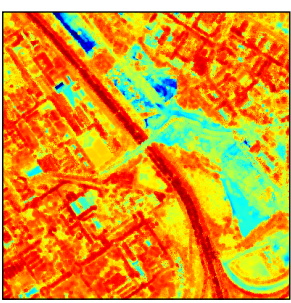

(d) Computed similarity

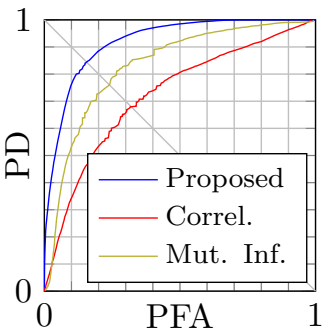

(f) Detection performance
Figure 5: Heterogeneous optical images before (a) and after (b) some construction work, with the corresponding change mask (c) and change map (d). The obtained estimated manifold (e) and detector performance (f).

sures linear dependency, whereas the mutual information captures a more general information-based dependency. Since both images correspond to the same kind of sensors and thus are linearly correlated, it is expected that the correlation coefficient and the mutual information perform similarly. This similar performance is observed in Fig. 4(f). Note that the proposed method is not based directly on statistical dependency between the pixel intensities. Instead, it is based on the dependency between the parameters of the image model presented in Section 2. We have observed that the proposed method shows good performance for homogeneous areas, contrary to the correlation coefficient and the mutual information, which explains its better performance.

\subsection{Optical images from heterogeneous sensors}

The second set of experiments aims at evaluating the performance of the proposed change detector for heterogeneous optical sensors. For this evaluation, we used a pair of Pléiades and Google Earth images. Both images are optical but were acquired using sensors with different specifications, i.e., different resolutions, different numbers of spectral bands, different central frequencies, etc. The image presented in Fig. 5(a) is the same as in Fig. 4(a), while the image in Fig. 5(b) corresponds to a Google Earth image of the same area after the beginning



(a) Google Earth - Dec. 2006

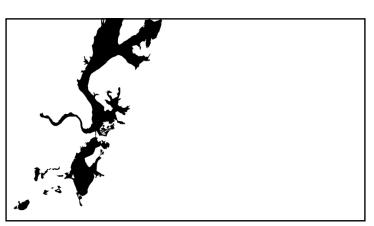

(c) Change mask

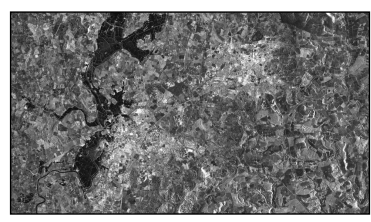

(b) TerraSAR-X - July 2007

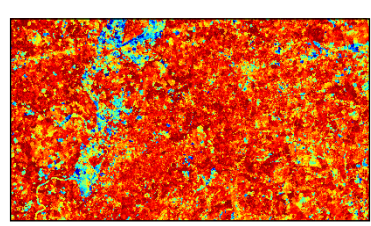

(d) Computed similarity

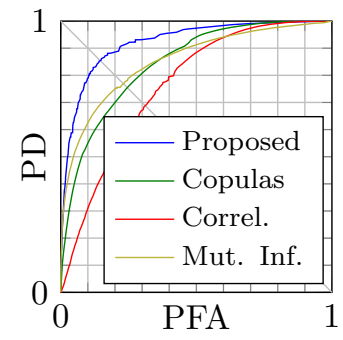

(e) Detection performance

Figure 6: Optical image before (a) and SAR image during (b) a flooding, with the corresponding change mask (c), change map (d) and resulting detection performance (e).

of building works. The Google Earth image has $3 \mathrm{spec}-$ tral bands and was resampled to match the $50 \mathrm{~cm}$ resolution of the Pléiades image. Fig. 5(c) shows the change mask between these two images used as a groundtruth. Finally, Fig. 5(d) shows the detection map obtained with the proposed change detection map with estimation windows of $40 \times 40$ pixels. The main changes due to building works can be observed in this detection map.

Fig. 5(e) shows a 2D projection of the estimated 7dimensional (4 dimensions for the Pléiades image and 3 dimensions for the Google Earth image) manifold. Since both images have been acquired by optical sensors, it is natural to observe some linear relationship for the two projected images. However, note that some non-linear components resulting from the sensor differences can also be observed.

Fig. 5(f) compares the performance of the proposed method with the detectors based on the correlation coefficient and the mutual information, which were also computed with windows of $40 \times 40$ pixels. It can be observed that the correlation coefficient performance decreases dramatically when compared to Fig. 4(f). This reduced performance of the correlation coefficient can be easily explained by the existence of the non-linear components in the manifold, revealing the non-linear relationships between the two images. However, the mutual information and the proposed methods remain both unaffected.

\subsection{Heterogeneous optical and SAR images}

The third experiments evaluates the performance of the proposed method for heterogeneous images. More 


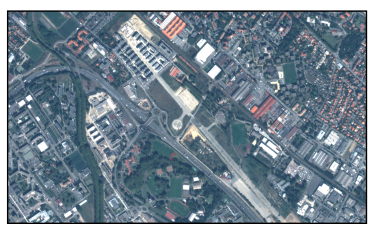

(a) Pléiades - July 2013

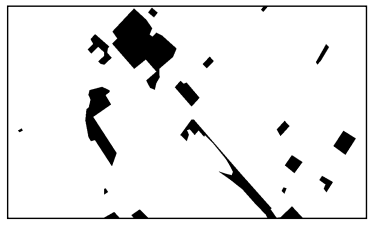

(c) Change mask

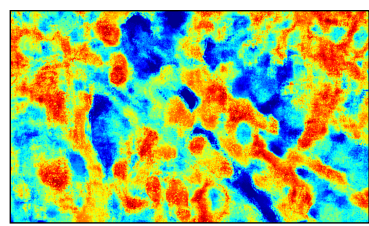

(e) Computed similarity

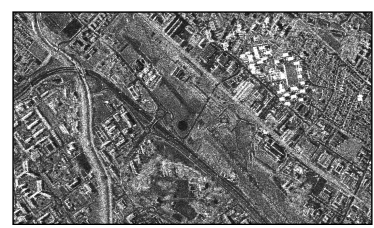

(b) TerraSAR-X - Feb 2009

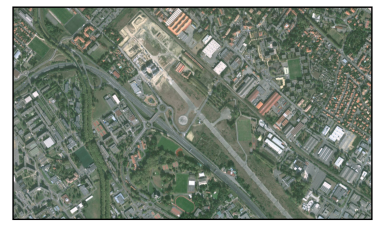

(d) Google Earth - Oct 2009

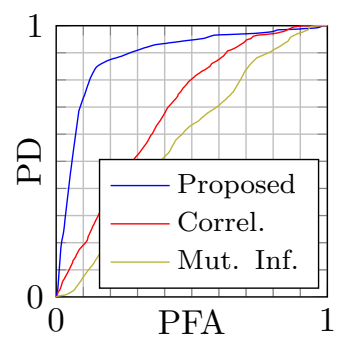

(f) Detection performance
Figure 7: Homogeneous optical images before (a) and after (b) construction works, and its change mask (c). Computed change map (d), and performance (f).

precisely, we have considered a Google Earth (optical) image and TerraSAR-X (radar) image. The images presented in Figs. 6(a) and 6(b) were taken before and during the July 2007 flooding of Gloucester. The TerraSAR$X$ image presents a pixel resolution of $7.3 \mathrm{~m}$, and the Google Earth image was down-sampled to match this resolution. Fig. 6(c) shows the change mask between these images representing the areas affected by the flooding. Fig. 6(d) shows the change detection map obtained with the proposed method when using a moving window of $10 \times 10$ pixels, covering an area of $73 \times 73 \mathrm{~m}^{2}$. The changes due to the flooding can be observed in this map.

The change detection performance obtained with the proposed method is shown in Fig. 6(e), along with the performance of other change detectors, including the approach detailed in (Mercier et al., 2008) ${ }^{2}$. A window size of $10 \times 10$ was used to compute the results obtained with the correlation coefficient and the mutual information. The results obtained with the algorithm based on conditional copulas was computed with a window size of $9 \times 9$ pixels. These results show that the performance of the proposed method is very interesting and that it is not really sensible to the fact that the images have been acquired by different kinds of sensors.

\subsection{Heterogeneous Pléiades and SAR images}

The last experiments are related to heterogeneous images, namely a set of higher resolution Pléiades and TerraSAR-X images. The images displayed in Figs. 7(a)

\footnotetext{
${ }^{2}$ The authors would like to thank Grégoire Mercier for providing the results obtained with the conditional copulas.
}

and 7 (b) were taken with an interval of 4 years in the South of Toulouse in France. The image in Fig. 7(d) was acquired just after the SAR image and is shown here to facilitate the visualization of the different changes, where some new constructions can be observed with respect to Fig. 7(a). Fig. 7(c) shows the change mask between these images. As in the previous tests, the image registration as well as the change mask were provided by a photo interpreter. Note that the optical image corresponds to the multi-spectral component of the Pléiades image, with a pixel resolution of $2 \mathrm{~m}$. The TerraSAR-X image was resampled accordingly to match the optical image. Fig. $7(\mathrm{e})$ shows the change map of our similarity measure and with a window size of $40 \times 40$ pixels.

The performance of the different detectors can be evaluated by the ROCs displayed in Fig. 7(f). Note that the same window sizes were used for detectors based on the correlation coefficient and the mutual information. This dataset appears to be difficult to be handled by the correlation coefficient and the mutual information. Conversely, the performance of the proposed method remains mostly unchanged when compared to the results obtained with the other datasets.

\section{Conclusions}

This paper investigated the performance of a statistical model introduced in (Prendes et al., 2014, 2015) for the detection of changes in co-registered images acquired by homogeneous or heterogeneous sensors. This model assumes that the image intensities located within an analysis windows are distributed according to a mixture of distributions defined by the noise statistics and the physical properties of the objects located in this analysis windows. The parameters of this mixture distribution are used to build a sensor linking vector that belongs to a manifold when there is no change between the images to be processed. A similarity measure can then be defined based on the distance between the sensor linking vector and the estimated manifold learned from training samples belonging to unchanged areas of the images. This similarity measure is finally used to as a test statistics for change detection. The main objective of this paper was to assess the performance of this change detection strategy for real optical (Google Earth and Pléiades) and SAR (TerraSAR-X) images. Based on the results obtained in this paper, we can conclude that this change detection strategy is very robust to the nature of the images to be analyzed. In particular, the detection performance was shown to be very promising for various scenarios including pairs of Pléiades images, Pléiades and Google Earth images and finally Pléiades and TerraSAR-X images. It is worth noting that all the images should be co-registered, which can be achieved in satellite images through the geolocation metadata provided by the satellite. Modifying the method in order to account for misregistration errors is clearly an interesting prospect. Future work also includes the study of a Bayesian nonparametric algorithm allowing the number of objects contained in the analysis windows to be estimated automatically. 


\section{References}

Carrara, W. G., Goodman, R. S., Majewski, R. M., 1995. Spotlight Synthetic Aperture Radar: Signal Processing Algorithms. Artech House signal processing library. Artech House.

Curlander, J. C., McDonough, R. N., 1991. Synthetic Aperture Radar: Systems and Signal Processing. Wiley Series in Remote Sensing and Image Processing. Wiley.

Figueiredo, M. A. T., Jain, A. K., March 2002. Unsupervised learning of finite mixture models. IEEE Trans. Pattern Anal. Mach. Intell. 24 (3), 381-396.

Mercier, G., Moser, G., Serpico, S. B., May 2008. Conditional copulas for change detection in heterogeneous remote sensing images. IEEE Trans. Geosci. and Remote Sensing 46 (5), 1428-1441.

Peterson, W. W., Birdsall, T., Fox, W., Sept. 1954. The theory of signal detectability. IRE Trans. Inf. Theory 4 (4), 171-212.

Poulain, V., Inglada, J., Spigai, M., Tourneret, J.-Y., Marthon, P., 2010. High resolution optical and SAR image fusion for road database updating. In: Proc. IEEE Int. Geosci. Remote Sens. Symp. (IGARSS). Honolulu, USA, pp. 2747-2750.

Prendes, J., Chabert, M., Pascal, F., Giros, A., Tourneret, J.Y., May 2014. A multivariate statistical model for multiple images acquired by homogeneous or heterogeneous sensors. In: Proc. IEEE Int. Conf. Acoust., Speech, and Signal Proc. Florence, Italy.

Prendes, J., Chabert, M., Pascal, F., Giros, A., Tourneret, J.-Y., March 2015. A new multivariate statistical model for change detection in images acquired by homogeneous and heterogeneous sensors. IEEE Trans. Image Process. 24 (3), 799-812.

Schowengerdt, R., 2006. Remote Sensing: Models and Methods for Image Processing. Elsevier Science.

Storie, C. D., Storie, J., Salinas de Salmuni, G., 2012. Urban boundary extraction using 2-component polarimetric SAR decomposition. In: Proc. IEEE Int. Geosci. Remote Sens. Symp. (IGARSS). Munich, Germany, pp. 5741-5744.

Thomas, C., Ranchin, T., Wald, L., Chanussot, J., May 2008. Synthesis of multispectral images to high spatial resolution: A critical review of fusion methods based on remote sensing physics. IEEE Trans. Geosci. and Remote Sensing 46 (5), 1301-1312.

Tison, C., Nicolas, J.-M., Tupin, F., Maitre, H., Oct. 2004. A new statistical model for Markovian classification of urban areas in high-resolution SAR images. IEEE Trans. Geosci. and Remote Sensing 42 (10), 2046-2057.

Uprety, P., Yamazaki, F., 2012. Use of high-resolution SAR intensity images for damage detection from the 2010 Haiti earthquake. In: Proc. IEEE Int. Geosci. Remote Sens. Symp. (IGARSS). Munich, Germany, pp. 6829-6832. 